\title{
Effects of near-source coagulation of biomass burning aerosols on global predictions of aerosol size distributions and implications for aerosol radiative effects
}

\section{Emily Ramnarine et al.}

Correspondence to: Emily Ramnarine (emily.ramnarine@ colostate.edu)

The copyright of individual parts of the supplement might differ from the CC BY 4.0 License. 
Fires in the Western US on 15/8/2015
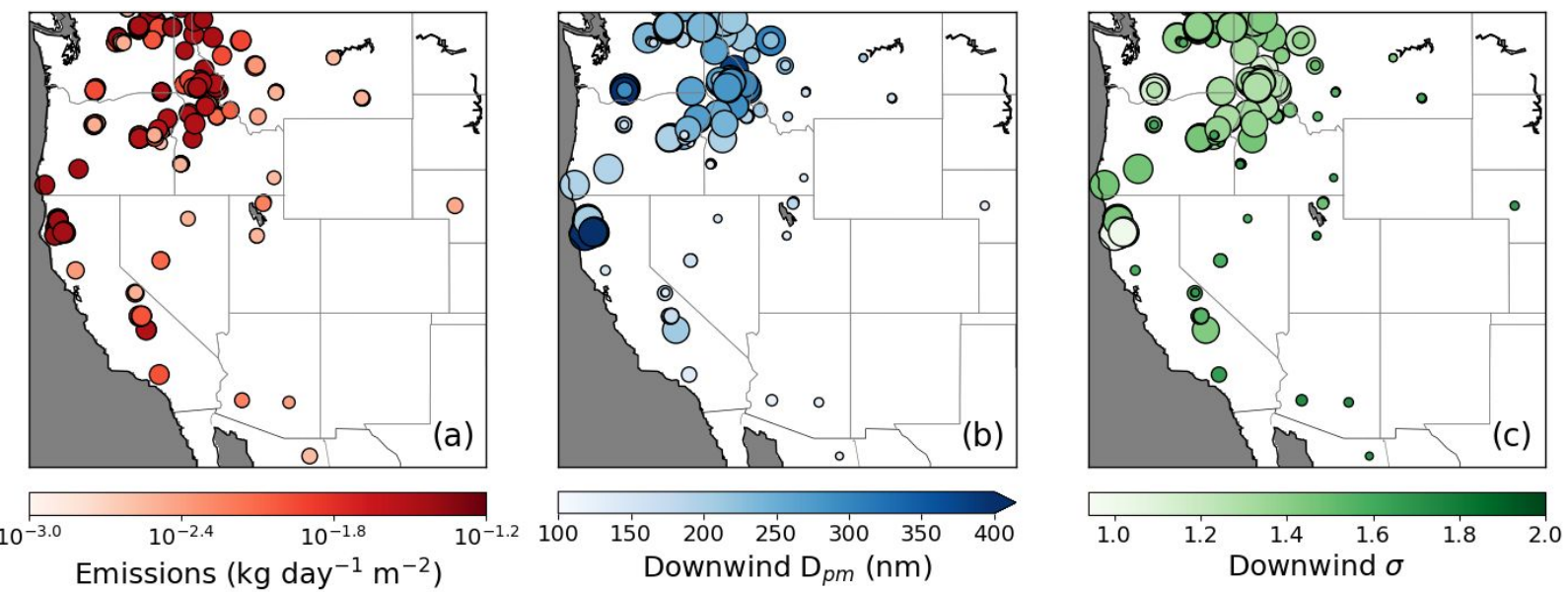

Figure S1: Demonstration of the effect of per-fire coagulation parameterization on all the fires in the Western US on 15 August 2015 from FINNv1.5. The emissions of $\mathrm{OA}$ and $\mathrm{BC}$ are indicated in the color in (a) and in the size of the markers in (b) and (c). In (a) the size indicated the area of the fire. In (b) and (c) the color indicates the median diameter and modal width, respectively, after 24 hours of coagulation where the emitted median diameter is $100 \mathrm{~nm}$ and the emitted modal width is 2 . 

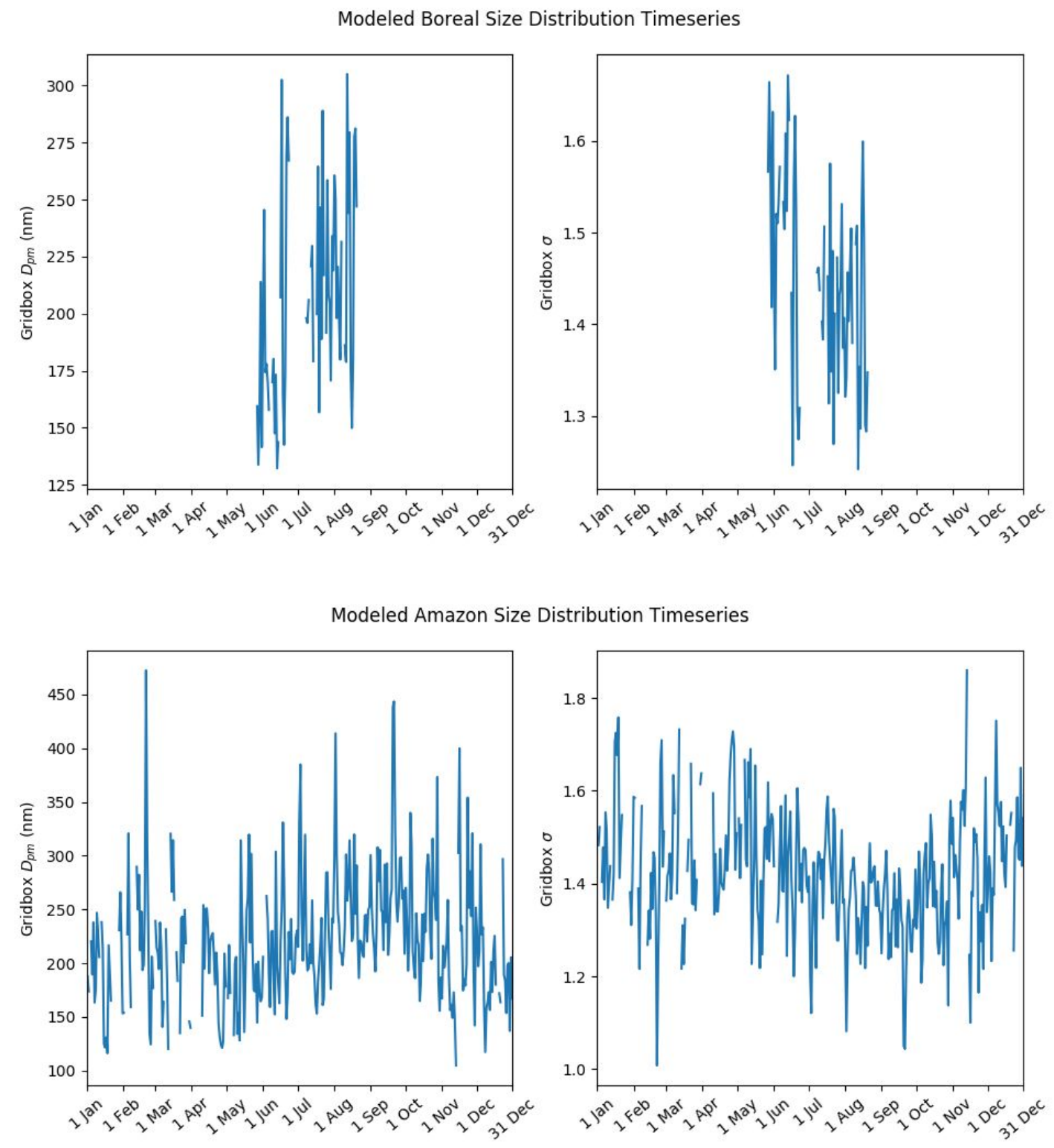

Figure S2: Daily temporal evolution of the predicted grid-resolved median diameter $\left(D_{\mathrm{pm}}\right.$; left) and modal width ( $\sigma$; right) in a box that spans $8^{\circ}$ latitude and $10^{\circ}$ for biomass burning emissions predicted for 2010 using FINNv1.5 fire emissions and the Sakamoto et al. (2016) parameterization after 24 hours of sub-grid coagulation with an emitted initial median diameter of $100 \mathrm{~nm}$ and an emitted initial modal width of 2 . The top plots are centered over Alaska at $62^{\circ} \mathrm{N}, 135^{\circ} \mathrm{W}$. Times that are not shown have no fire data in this gridbox. The bottom plots are centered over the Amazon at $6^{\circ} \mathrm{S}, 60^{\circ} \mathrm{W}$. 

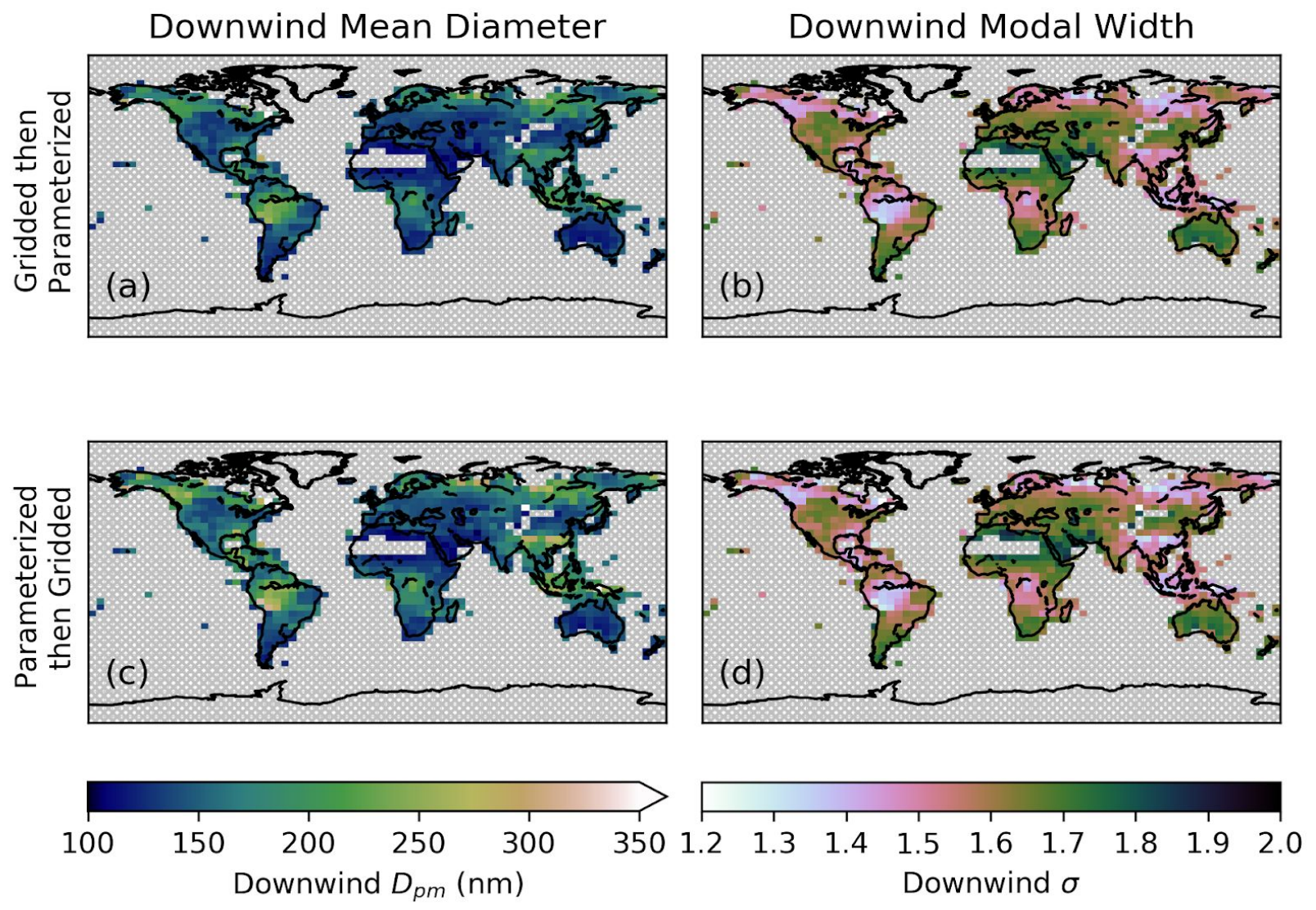

Figure S3: Annual-mean median diameter (a and, c) and modal width (b and, d) for biomass burning emissions predicted for 2010 using the Sakamoto et al. (2016) parameterization after 24 hours of sub-grid coagulation with an emitted initial median diameter of $100 \mathrm{~nm}$ and an emitted initial modal width of 2 . Panels (a) and (b) show the resulting $D_{\mathrm{pm}}$ and $\sigma$ when the fire (FINNv1.5) and meteorological data is averaged over a $4^{\circ} \times 5^{\circ}$ grid and then that gridded data is run through the Sakamoto et al. (2016) parameterization. Panels (c) and (d) show the results when the individual fires are run through the Sakamoto et al. (2016) parameterization and then the output Dpm and $\sigma$ are averaged over a $4^{\circ} \times 5^{\circ}$ grid. The regions with grey crosshatching are grid-cells with no fire data. 


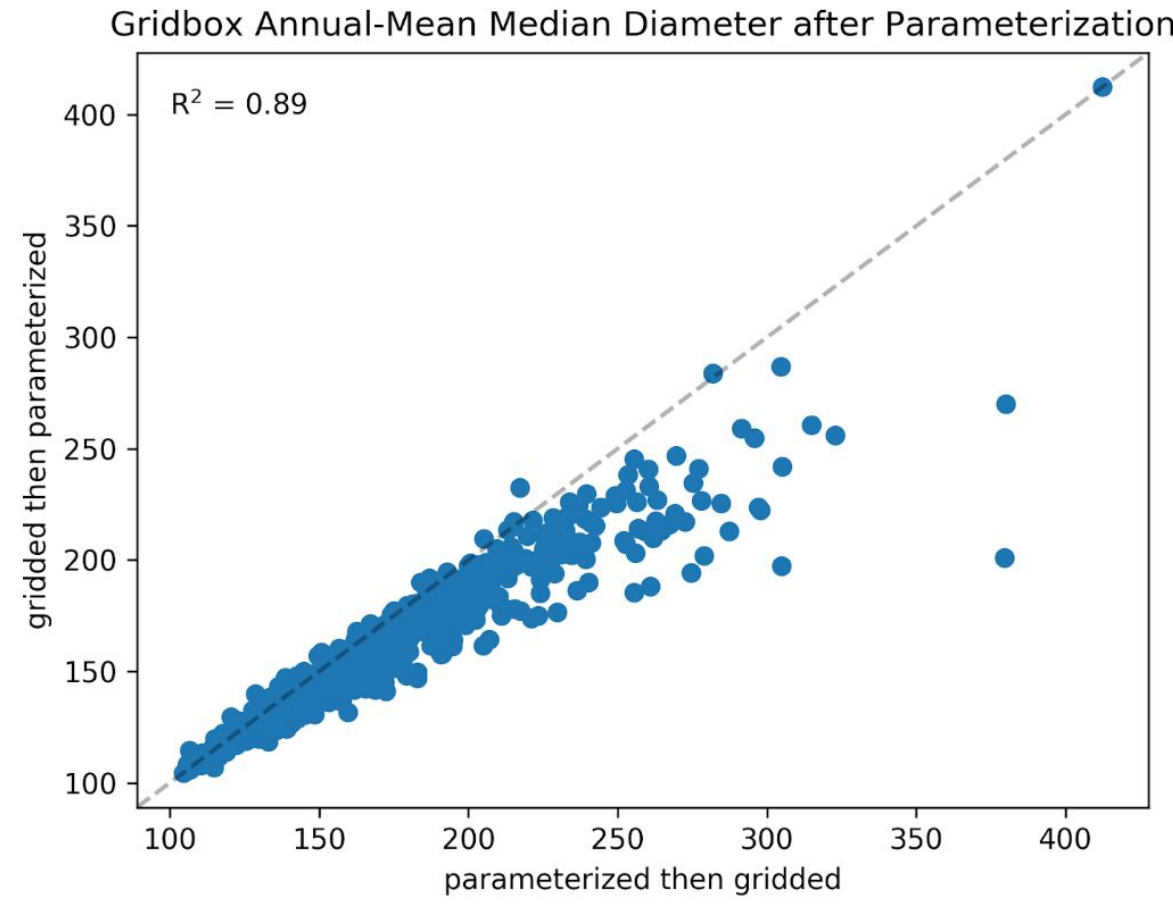

Figure S4: Annual-mean median diameter for biomass burning emissions predicted for 2010 using the Sakamoto et al. (2016) parameterization after 24 hours of sub-grid coagulation with an emitted initial median diameter of $100 \mathrm{~nm}$ and an emitted initial modal width of 2 . The y-axis shows the resulting median modal width when the fire (FINNv1.5) and meteorological data is averaged over a $4^{\circ} \times 5^{\circ}$ grid and then that gridded data is run through the Sakamoto et al. (2016) parameterization (what we use in GEOS-Chem in this study). The $\mathrm{x}$-axis shows the results when the individual fires are run through the Sakamoto et al. (2016) parameterization and then the output median modal width is averaged over a $4^{\circ} \times 5^{\circ}$ grid. Each gridbox globally is represented as a point on the plot. The 1:1 line is in grey dash. 


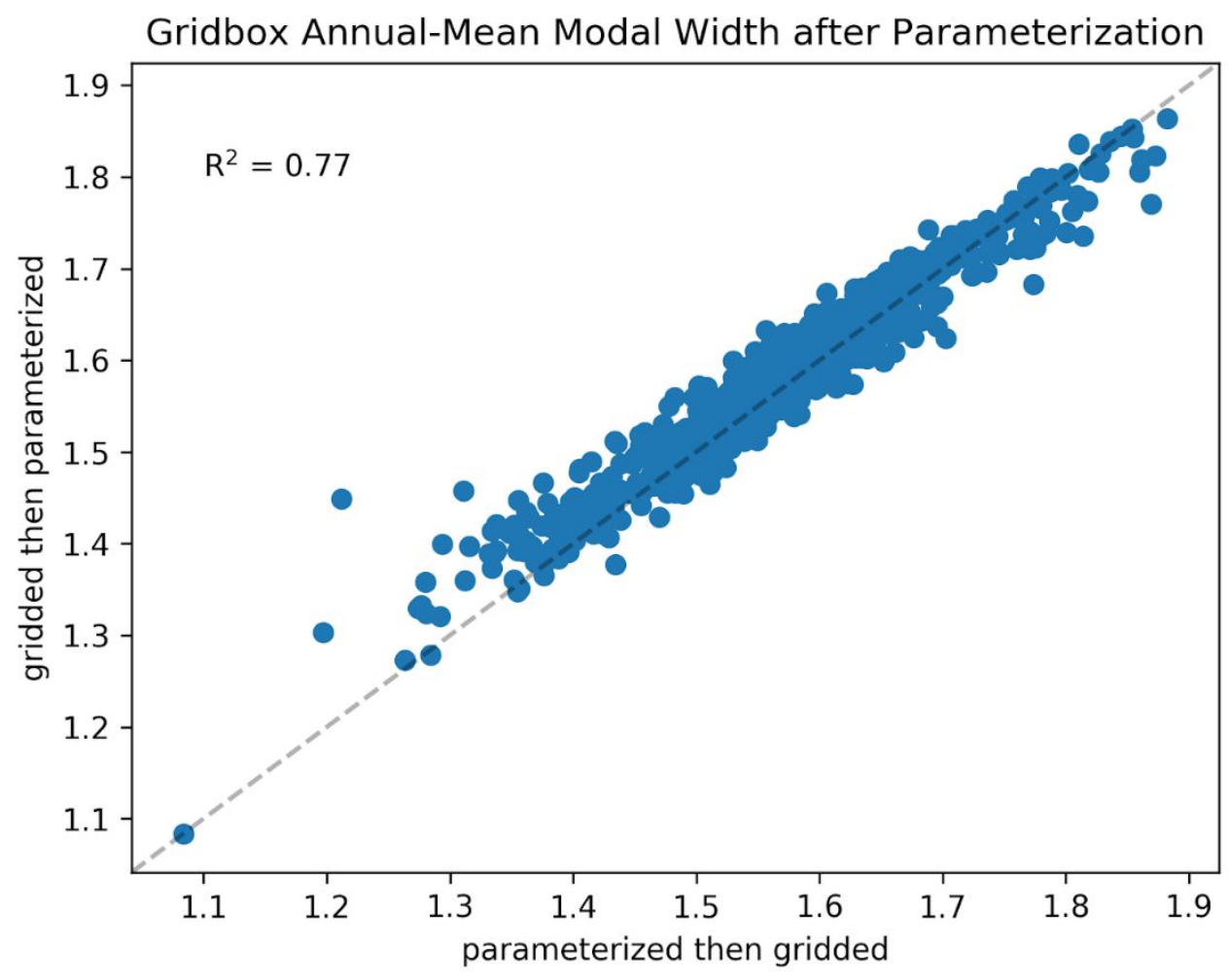

Figure S5: Annual-mean modal width for biomass burning emissions predicted for 2010 using the Sakamoto et al. (2016) parameterization after 24 hours of sub-grid coagulation with an emitted initial median diameter of $100 \mathrm{~nm}$ and an emitted initial modal width of 2 . The y-axis shows the resulting lognormal modal width when the fire (FINNv1.5) and meteorological data is averaged over a $4^{\circ} \times 5^{\circ}$ grid and then that gridded data is run through the Sakamoto et al. (2016) parameterization (what we use in GEOS-Chem in this study). The xaxis shows the results when the individual fires are run through the Sakamoto et al. (2016) parameterization and then the output lognormal modal width is averaged over a $4^{\circ} \times 5^{\circ}$ grid. Each gridbox globally is represented as a point on the plot. The 1:1 line is in grey dash. 

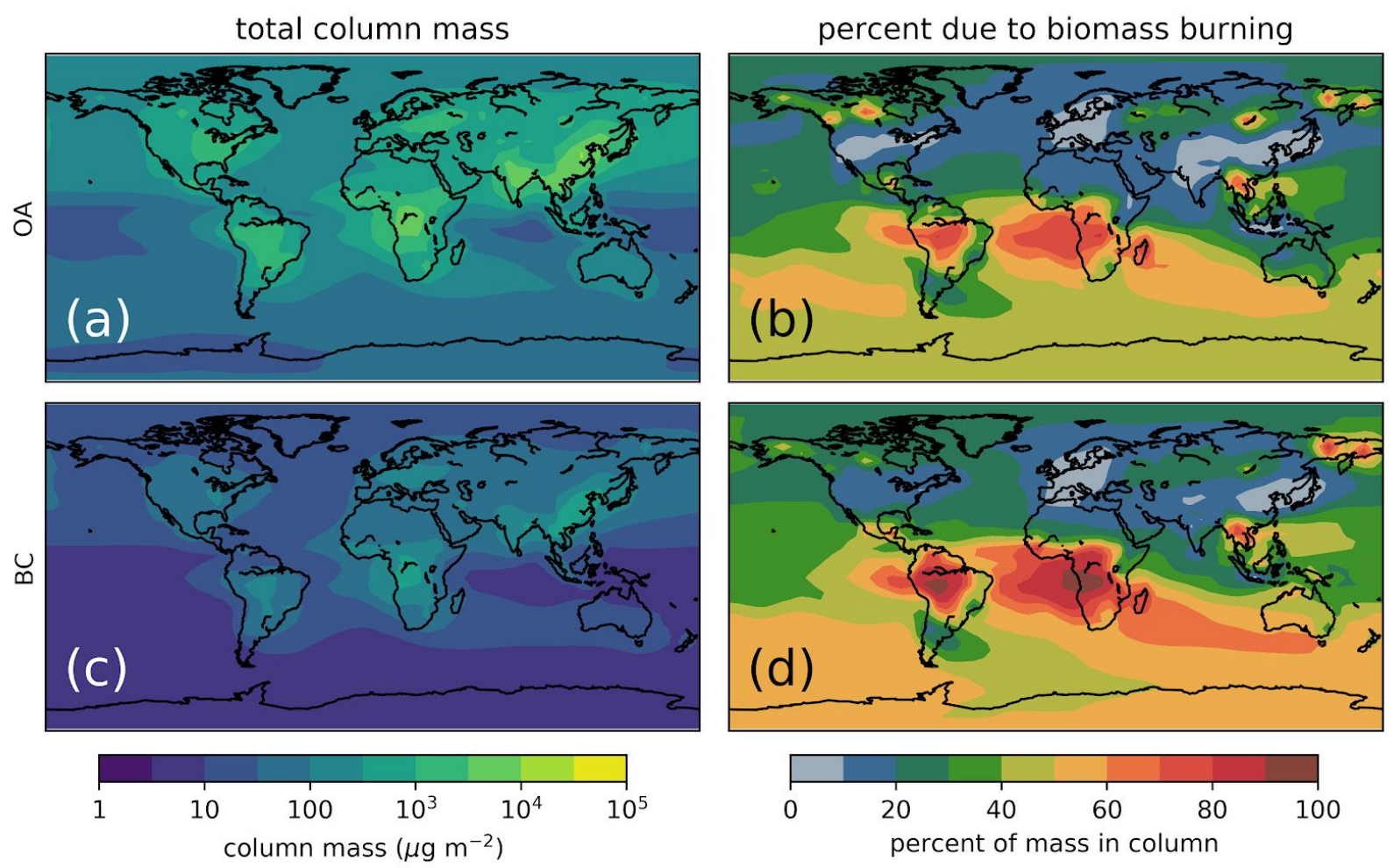

Figure S6: Effect of biomass burning on annually averaged total column $\mathrm{OA}$ and $\mathrm{BC}$ mass concentrations. The left side shows the total column mass concentration of (a) OA and (c) BC in the simulations with FINNv1 biomass burning emissions (i.e., noSubCoag_FINN). The right side shows the percent of the mass in the column that is due to biomass burning emissions for (b) $\mathrm{OA}$ and (d) $\mathrm{BC}$ by taking the difference between the noSubCoag_FINN and noBB simulations. 

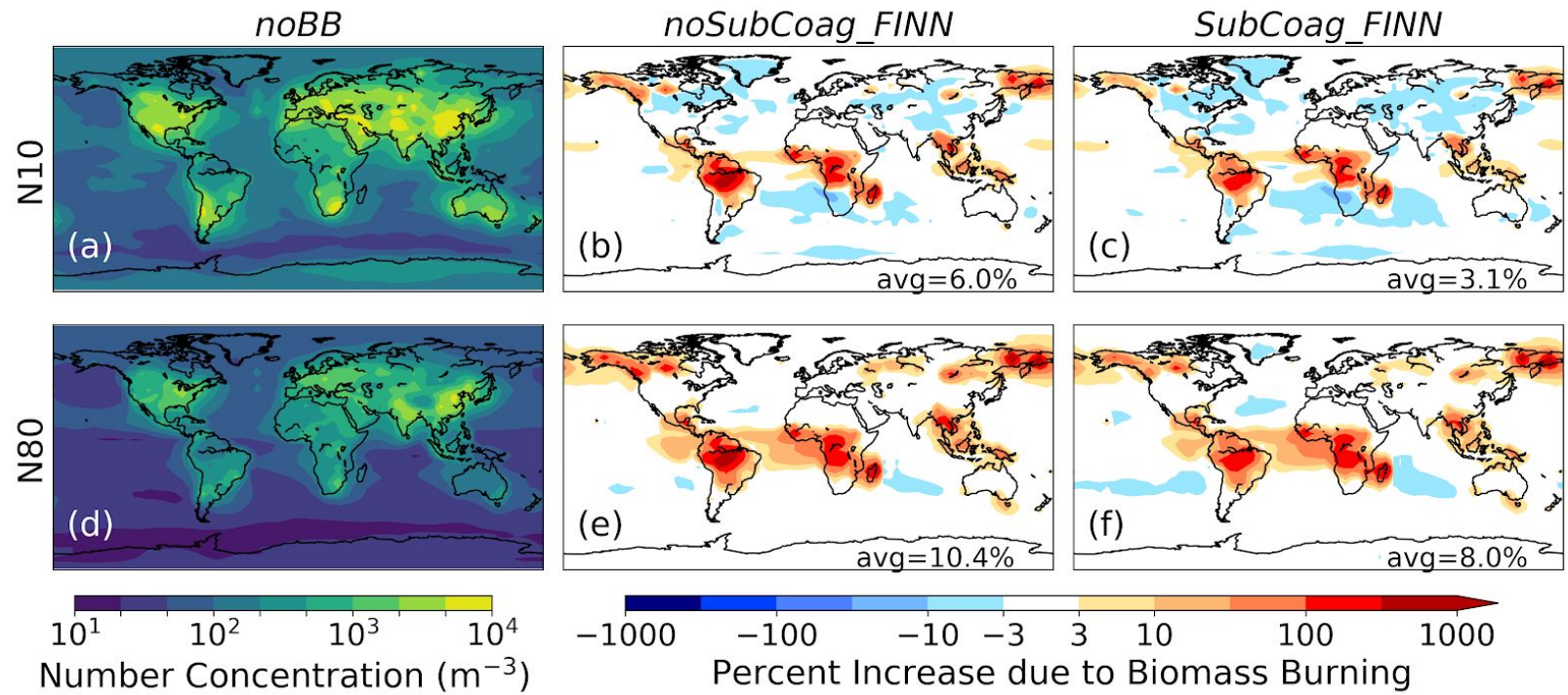

Figure S7: Effect of biomass burning on surface-level number concentration of particles above $10 \mathrm{~nm}(\mathrm{a}, \mathrm{b}, \mathrm{c})$ and $80 \mathrm{~nm}$ (d, e, f). Panels (a) and (d) show the absolute number concentration for the noBB simulation. Panels (b) and (e) show the percent increase due to FINNv1 biomass burning emissions from the $n o B B$ simulation to the noSubCoag_FINN simulation. Panels (c) and (f) show the percent increase due to FINNv1

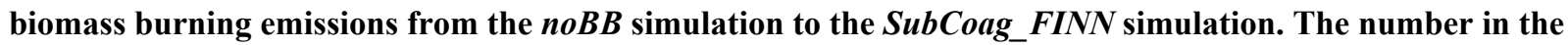
bottom right of each panel is the global mean percent increase due to biomass burning. 

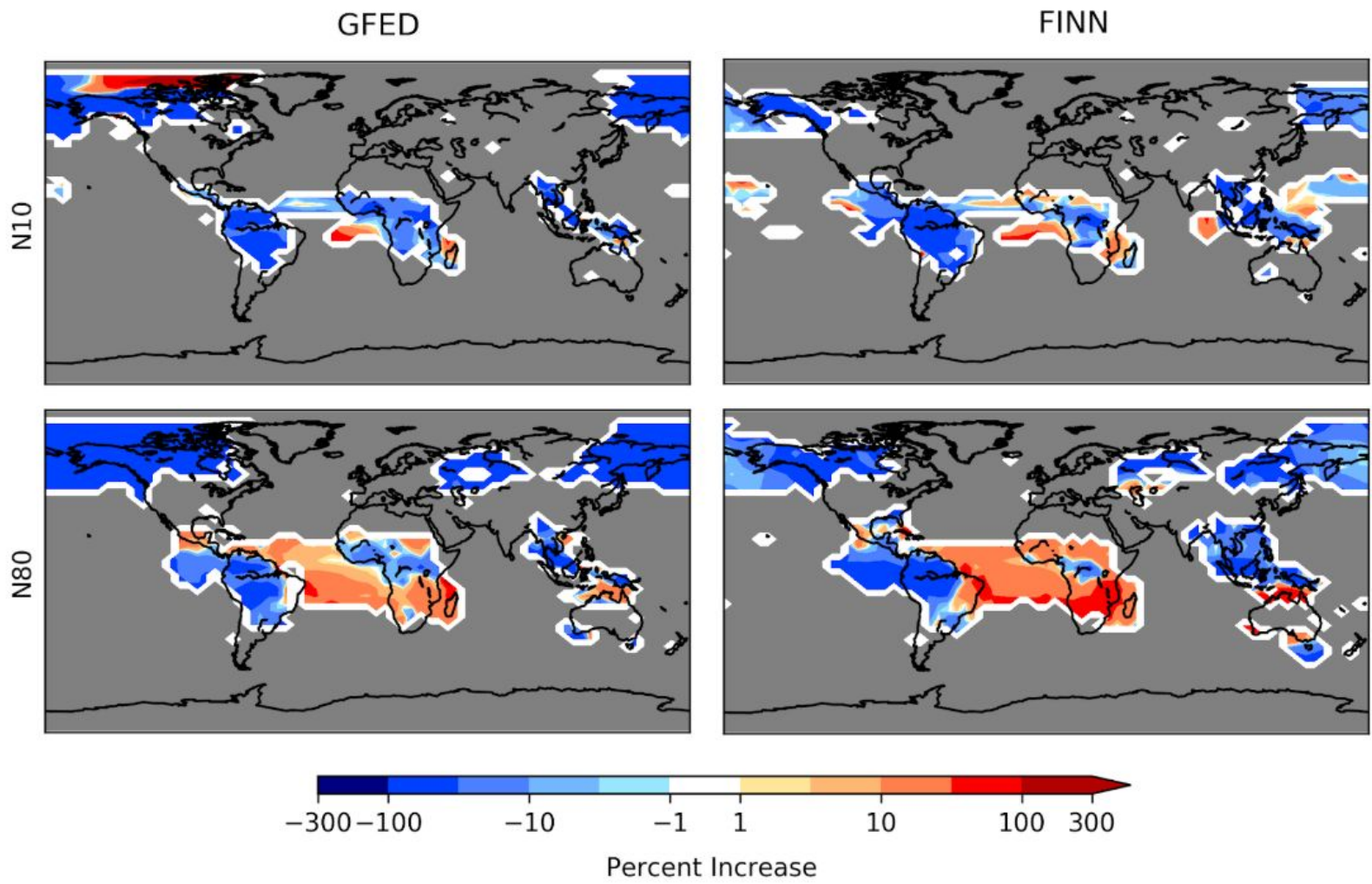

Figure S8: Percent change in the relative contribution of biomass burning to particles larger than $10 \mathrm{~nm}$ (N10) and particles larger than $80 \mathrm{~nm}$ (N80) due to sub-grid coagulation when using GFED emissions (left) or FINNv1 emissions (right), and an initial median diameter of $100 \mathrm{~nm}$, initial modal width of 2 , and coagulation time of 24 hours. Negative values correspond to a reduced impact of biomass burning when sub-grid coagulation is added. Regions where the percent increase of N10 or N80 due to biomass burning is less than $1 \%$ (with or without sub-grid coagulation, see Figs. 3 and S7) are shaded in grey. 

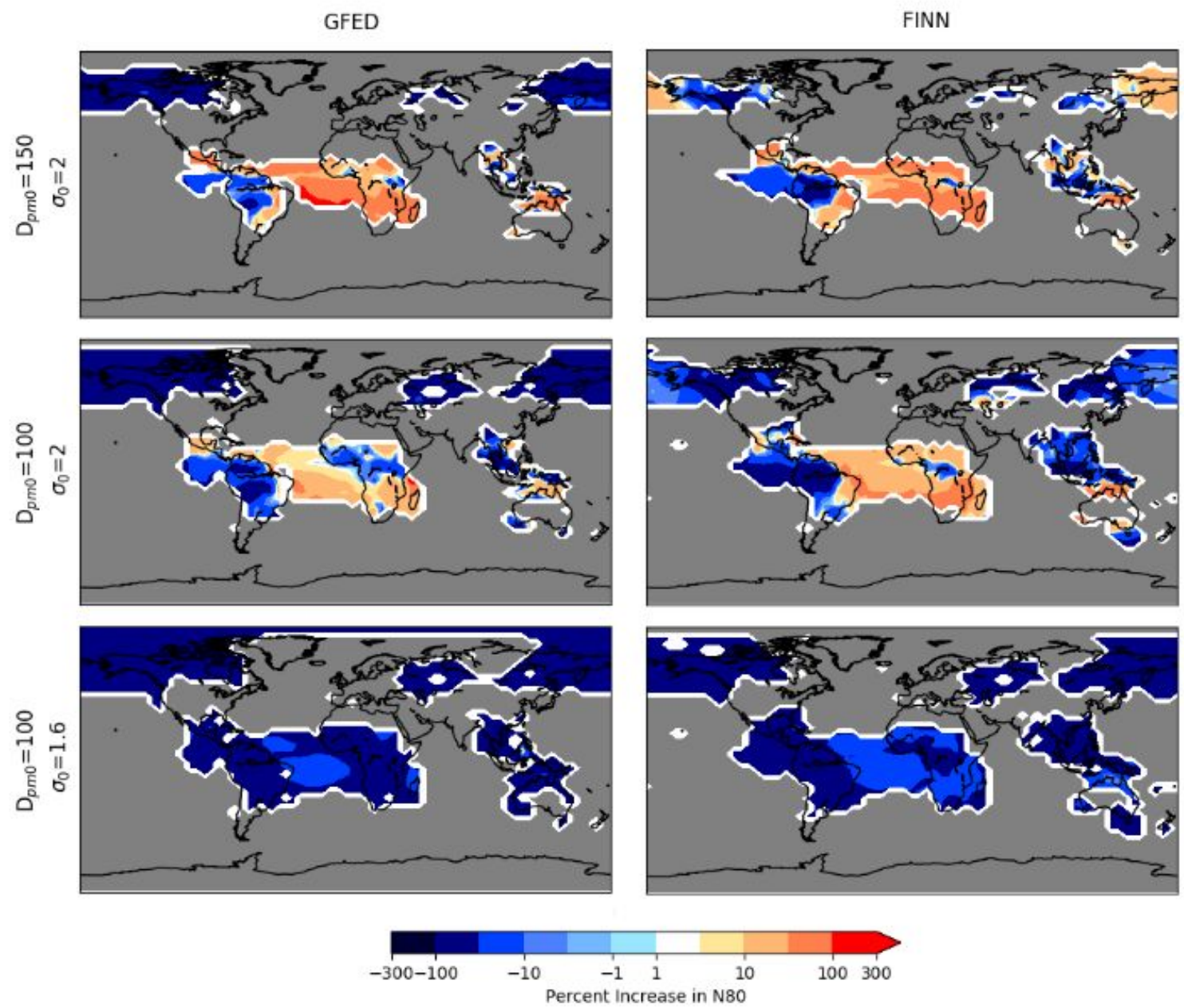

Figure S9: Percent change in the relative contribution of biomass burning to particles larger than $80 \mathrm{~nm}$ due to sub-grid coagulation when using GFED emissions (left) or FINNv1 emissions (right) and a coagulation time of 24 hours. Panels in the top row have an emitted median diameter of $150 \mathrm{~nm}$ and an emitted modal width of 2. Panels in the middle row have an emitted median diameter of $100 \mathrm{~nm}$ and an emitted modal width of 2. Panels in the bottom row have an emitted median diameter of $100 \mathrm{~nm}$ and an emitted modal width of 1.6. Negative values correspond to a reduced impact of biomass burning when sub-grid coagulation is added. Regions where the percent increase of $\mathrm{N80}$ due to biomass burning is less than $1 \%$ (with or without sub-grid coagulation, see Figs. 4 and S10) are shaded in grey. 


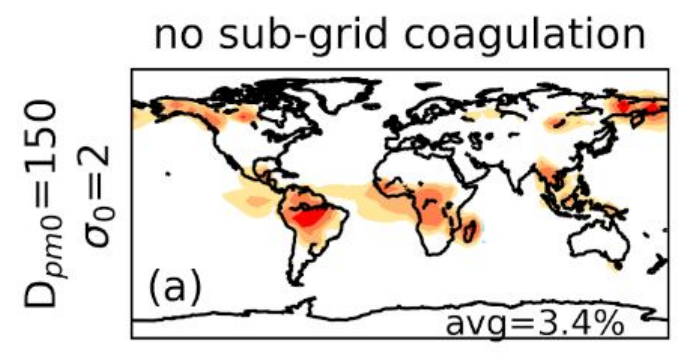

with sub-grid coagulation
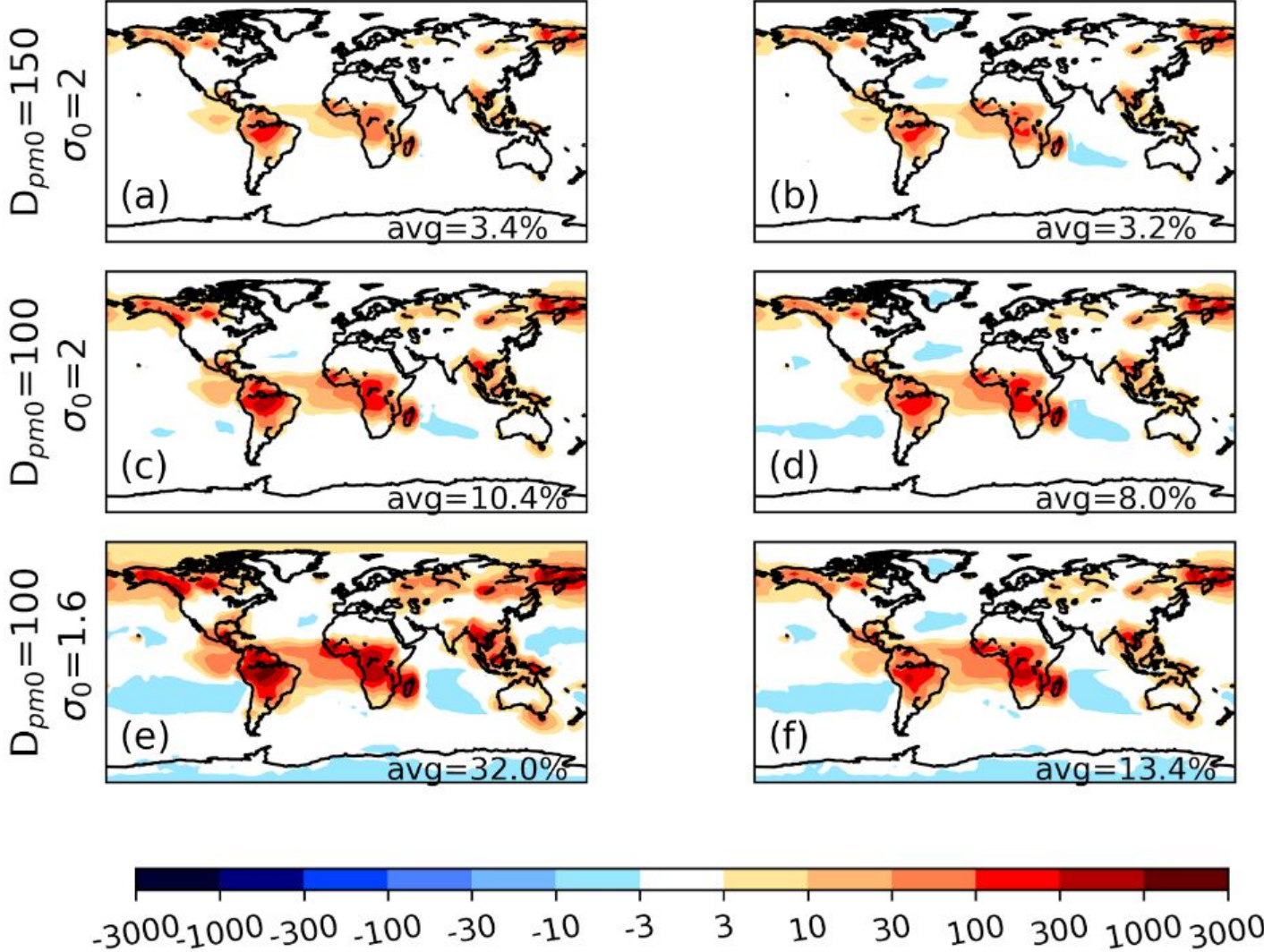

Percent Increase in N80 due to BB

Figure S10: Annual-average percent changes in N80 at the surface level due to the inclusion of FINNv1 biomass burning emissions relative to the simulation without biomass burning (noBB). Panels (a), (c), and (e) have no sub-grid coagulation (D150_noSubCoag_FINN, noSubCoag_FINN, and s1.6_noSubCoag_FINN, respectively). Panels (b), (d), and (f) have sub-grid coagulation (D150_SubCoag_FINN, SubCoag_FINN, and s1.6_SubCoag_FINN, respectively). Panels (a) and (b) have an emitted median diameter of $150 \mathrm{~nm}$ and an emitted modal width of 2. Panels (c) and (d) have an emitted median diameter of $100 \mathrm{~nm}$ and an emitted modal width of 2. Panels (e) and (f) have an emitted median diameter of $100 \mathbf{~ n m}$ and an emitted modal width of 1.6. The number in the bottom right of each panel is the global mean percent increase in N80 due to biomass burning. 

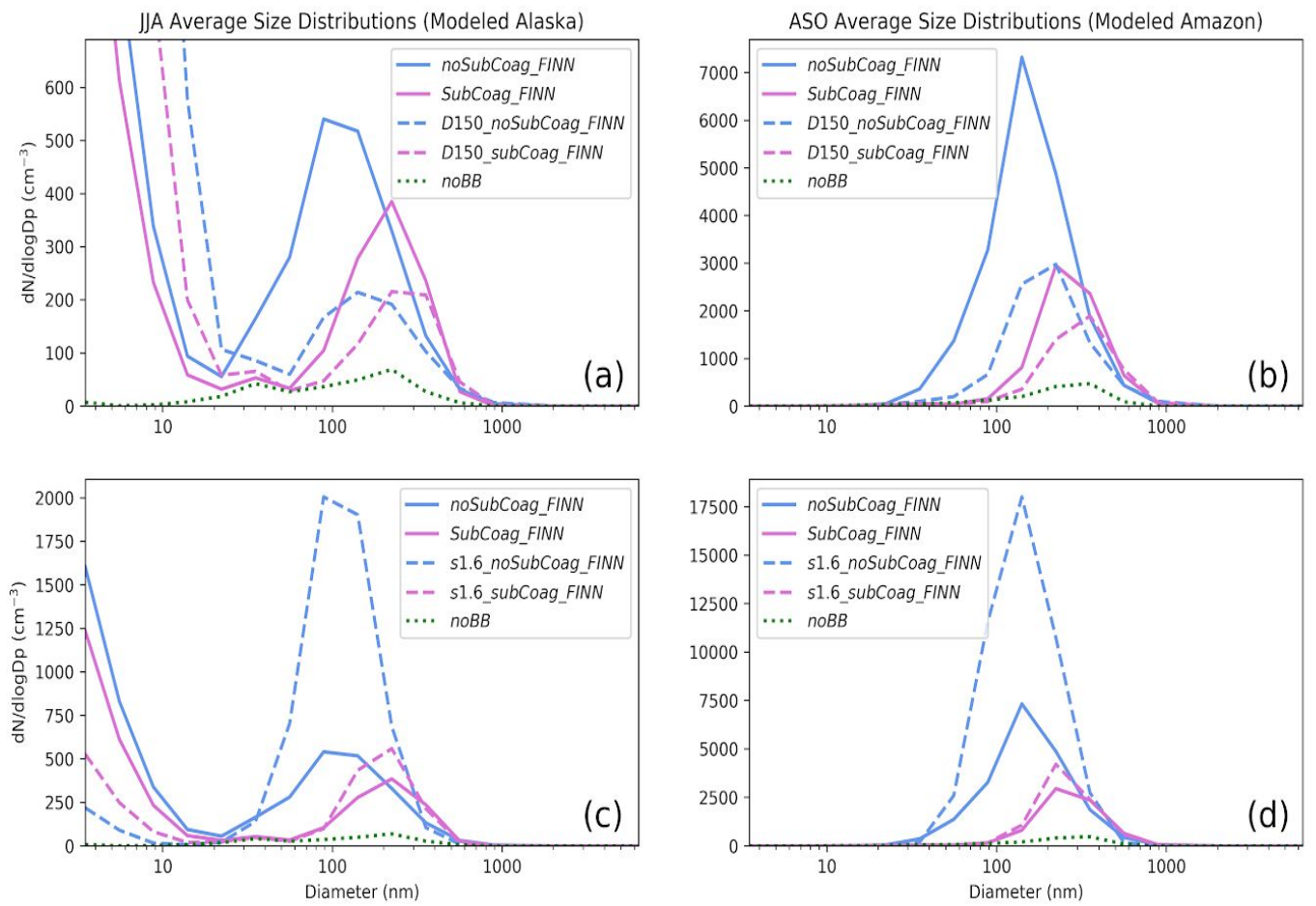

Figure S11: Predicted grid-resolved aerosol size distributions with FINNv1 biomass burning emissions over Alaska at $62^{\circ} \mathrm{N}, 140^{\circ} \mathrm{W}$, averaged over the June, July, and August fire season (a and c) and the Amazon at $6^{\circ}$ $\mathrm{S}, 60^{\circ} \mathrm{W}$, averaged over the August, September, and October fire season (b and d). All panels show the size distributions for the noBB, noSubCoag_FINN, and SubCoag_FINN simulations in the dashed green, solid blue, and solid pink lines, respectively. The top panels (a and b) show the sensitivity to the emitted median diameter, and the bottom panels (c and d) show the sensitivity to the emitted modal width. Note the different y-axis scales. 


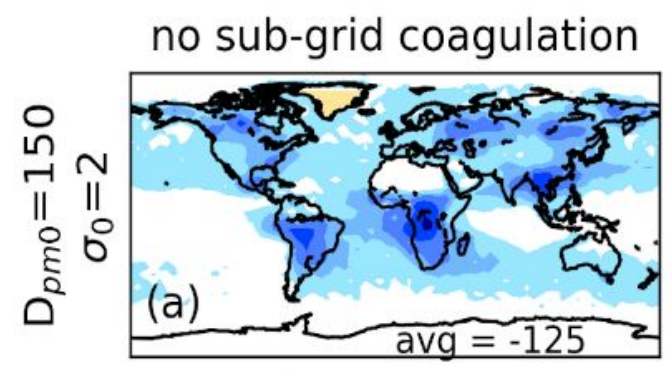

with sub-grid coagulation
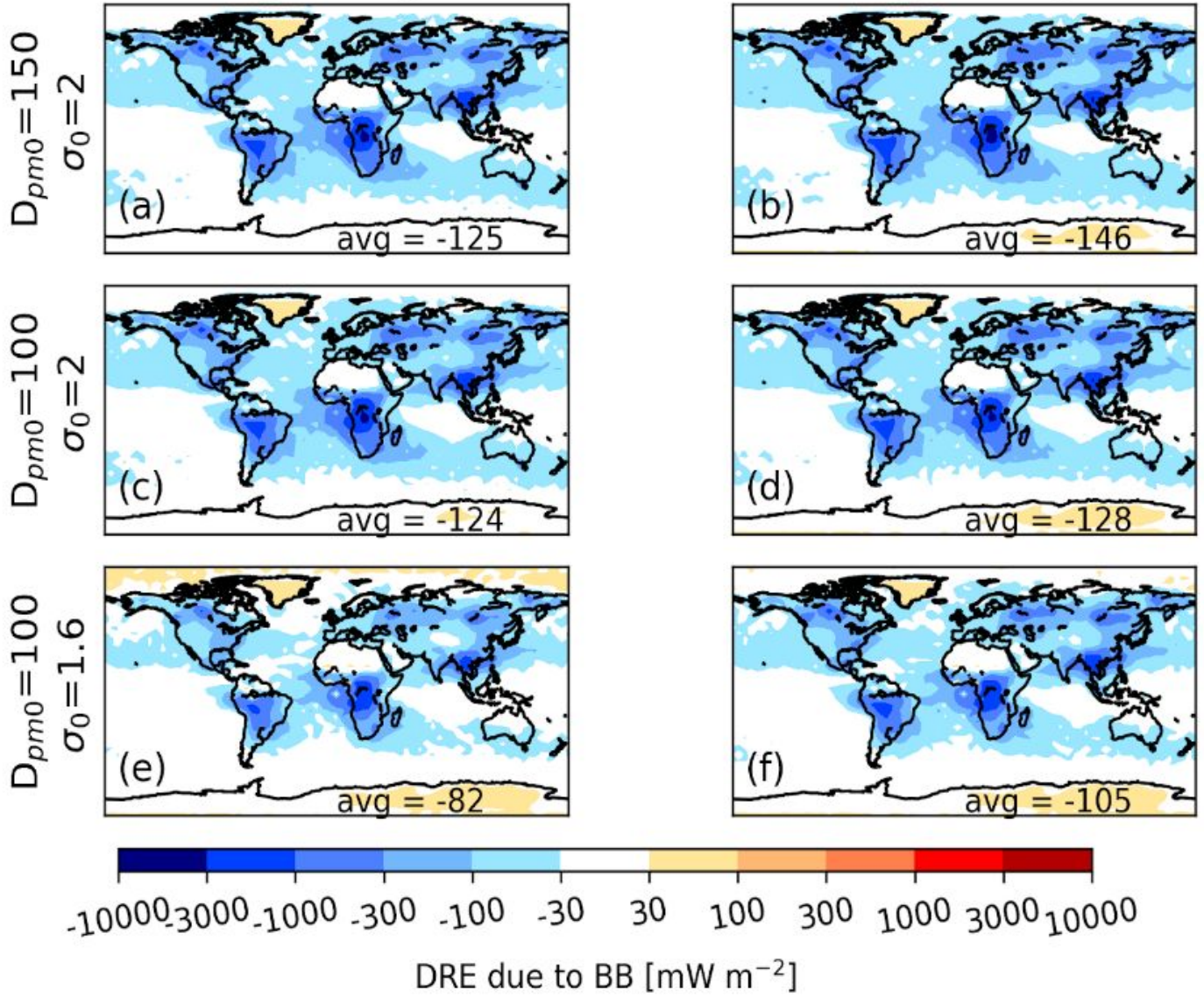

Figure S12: All-sky direct radiative effect (DRE) due to biomass burning aerosols using FINNv1 emissions and using the external-mixing assumption. Panels (a), (c), and (e) are without sub-grid coagulation (D150_noSubCoag_FINN, noSubCoag_FINN, and s1.6_noSubCoag_FINN, respectively). Panels (b), (d), and (f) are with sub-grid coagulation(D150_SubCoag_FINN,SSubCoag_FINN, and s1.6_SubCoag_FINN, respectively). Panels (a) and (b) have an emitted median diameter of $150 \mathrm{~nm}$ and an emitted modal width of 2. Panels (c) and (d) have an emitted median diameter of $100 \mathrm{~nm}$ and an emitted modal width of 2. Panels (e) and (f) have an emitted median diameter of $100 \mathrm{~nm}$ and an emitted modal width of 1.6. The number in the bottom right of each panel is the global mean DRE value $\left[\mathrm{mW} \mathrm{m}^{-2}\right]$. 
GFED
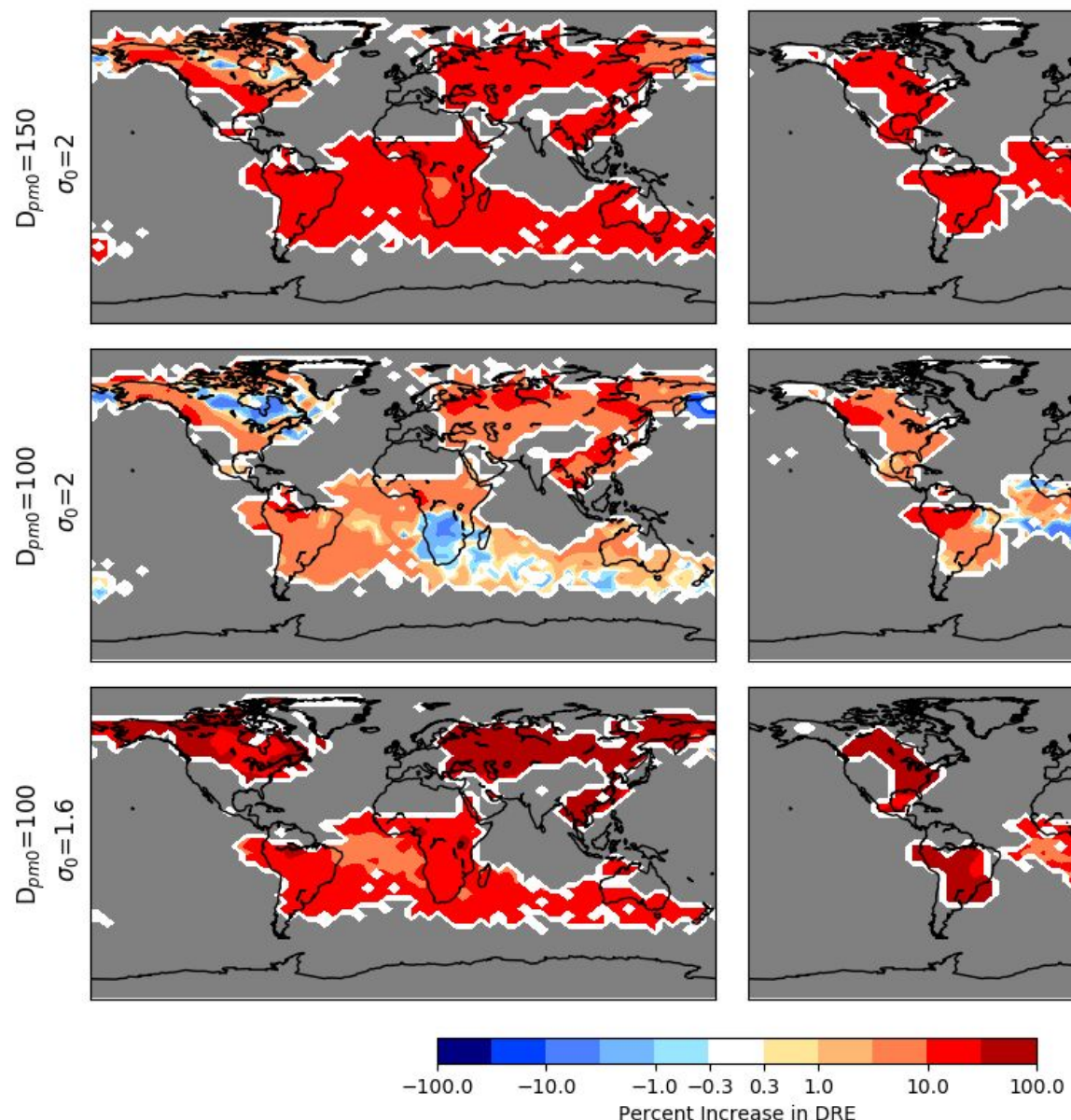

Figure S13: Percent change in all-sky direct radiative effect (DRE) of biomass burning aerosol due to subgrid coagulation when using the external mixing assumption and GFED emissions (left) or FINNv1 emissions (right) and an assumed in-plume coagulation time of 24 hours. Panels in the top row have an emitted median diameter of $150 \mathrm{~nm}$ and an emitted modal width of 2 . Panels in the middle row have an emitted median diameter of $100 \mathrm{~nm}$ and an emitted modal width of 2 . Panels in the bottom row have an emitted median diameter of $100 \mathrm{~nm}$ and an emitted modal width of 1.6. Positive (red) values correspond to an increased cooling tendency of biomass burning DRE due to sub-grid coagulation being added. Regions in grey indicate that the DRE due to biomass burning is a more positive value than $-100 \mathrm{~mW} \mathrm{~m}^{-2}$ (i.e. there is less than 100

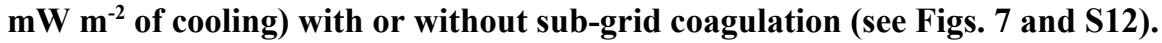




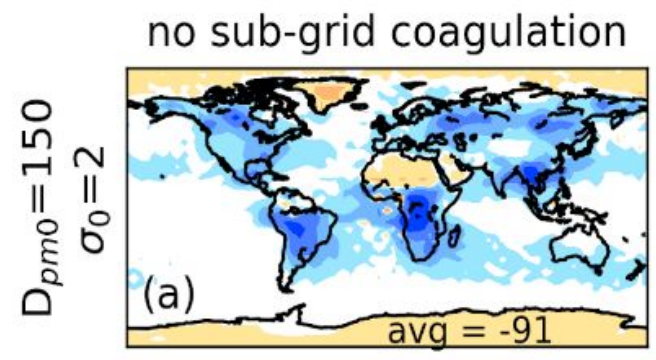

with sub-grid coagulation
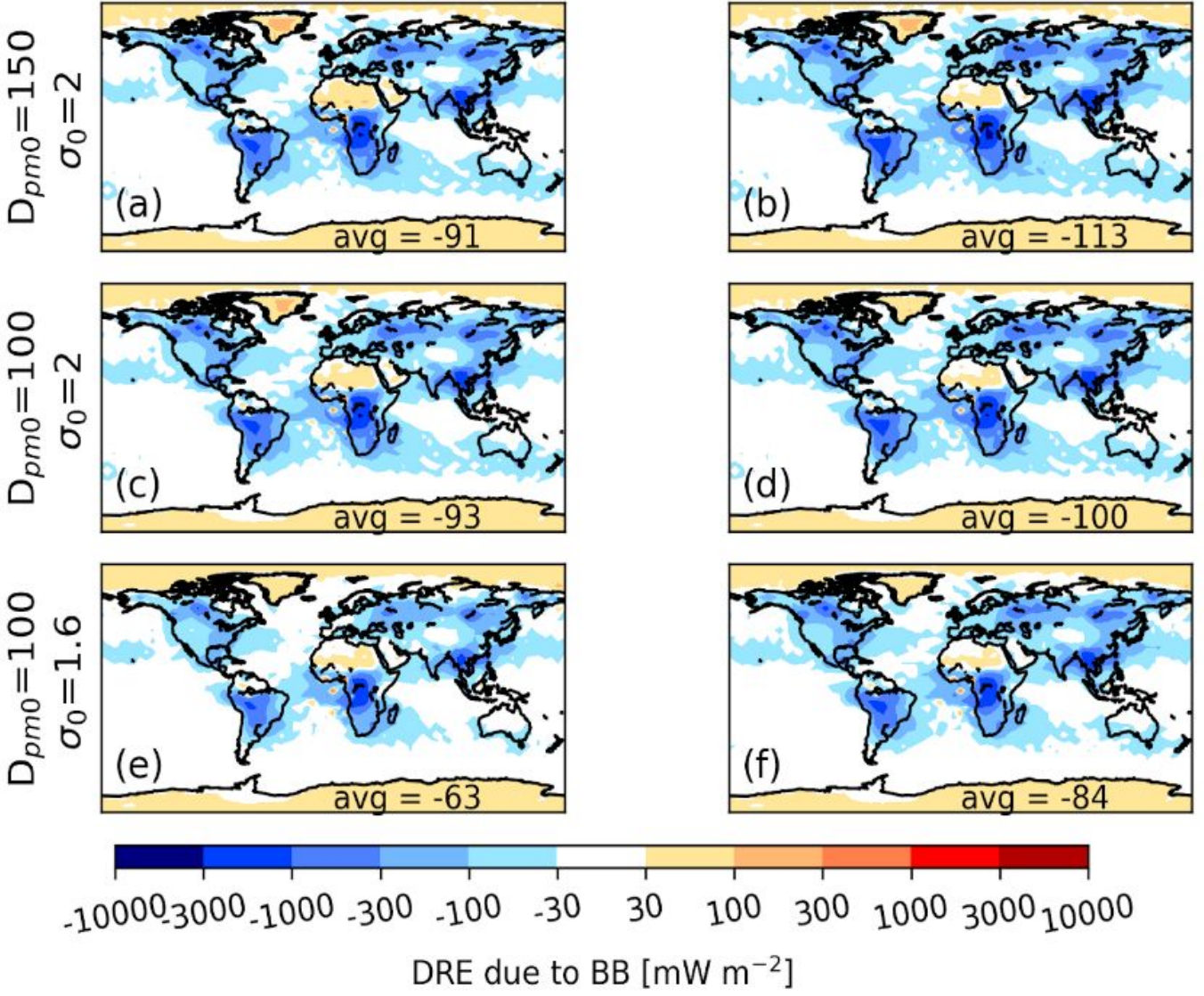

Figure S14: All-sky direct radiative effect (DRE) due to biomass burning aerosols using FINNv1 emissions and using the core-shell mixing assumption. Panels (a), (c), and (e) are without sub-grid coagulation (D150_noSubCoag_FINN, noSubCoag_FINN, and s1.6_noSubCoag_FINN, respectively). Panels (b), (d), and (f) are with sub-grid coagulation(D150_SubCoag_FINN,SUbCoag_FINN, and s1.6_SubCoag_FINN, respectively). Panels (a) and (b) have an emitted median diameter of $150 \mathrm{~nm}$ and an emitted modal width of 2. Panels (c) and (d) have an emitted median diameter of $100 \mathrm{~nm}$ and an emitted modal width of 2. Panels (e) and (f) have an emitted median diameter of $100 \mathrm{~nm}$ and an emitted modal width of 1.6. The number in the bottom right of each panel is the global mean DRE value $\left[\mathrm{mW} \mathrm{m}^{-2}\right]$. 

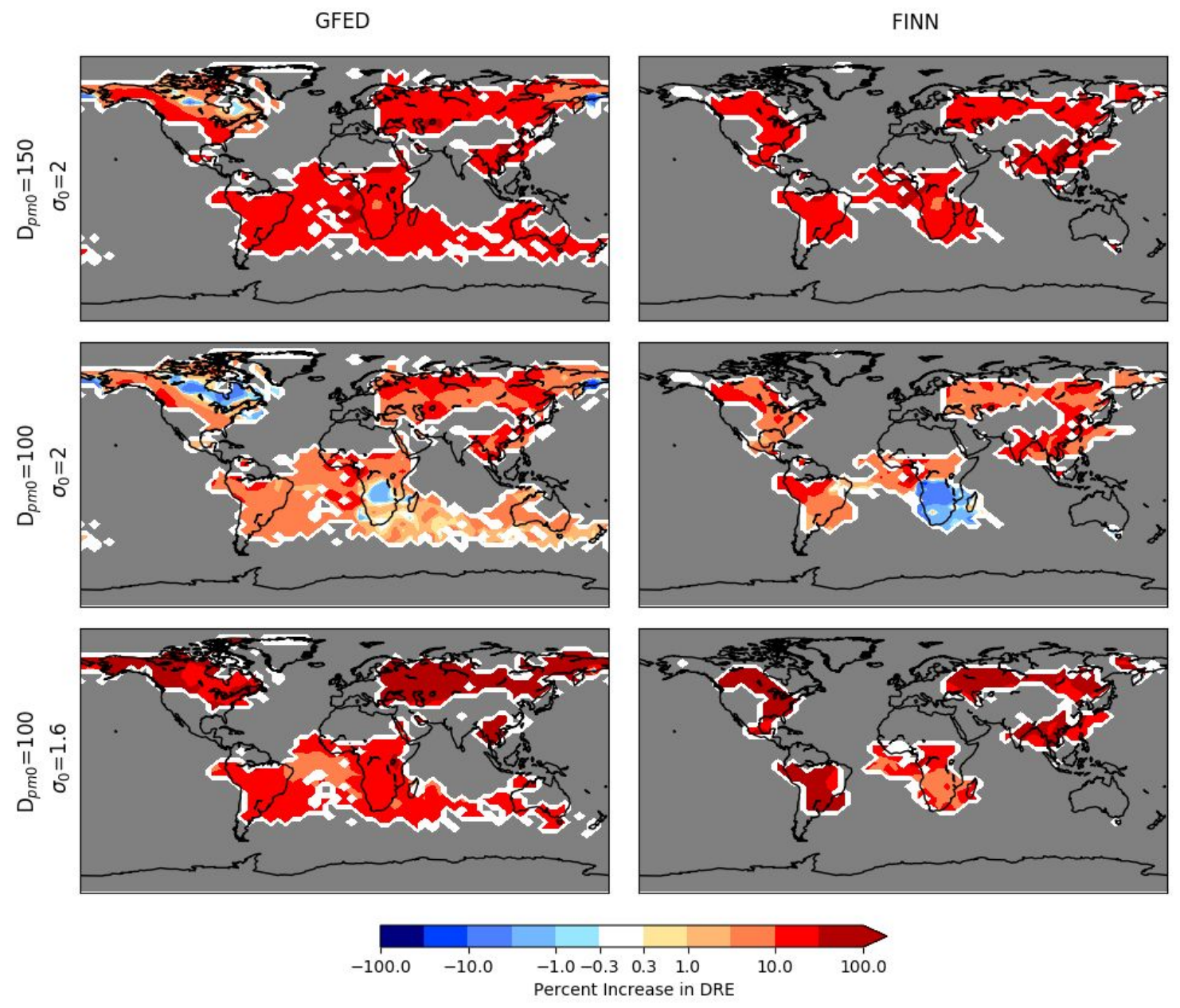

Figure S15: Percent change in all-sky direct radiative effect (DRE) of biomass burning aerosol due to subgrid coagulation when using the core-shell mixing and GFED emissions (left) or FINNv1 emissions (right) and an assumed in-plume coagulation time of 24 hours. Panels in the top row have an emitted median diameter of $150 \mathrm{~nm}$ and an emitted modal width of 2 . Panels in the middle row have an emitted median diameter of 100 $\mathrm{nm}$ and an emitted modal width of 2 . Panels in the bottom row have an emitted median diameter of $100 \mathrm{~nm}$ and an emitted modal width of 1.6. Positive (red) values correspond to an increased cooling tendency of biomass burning DRE due to sub-grid coagulation being added. Regions in grey indicate that the DRE due to biomass burning is a more positive value than $-100 \mathrm{~mW} \mathrm{~m}^{-2}$ (i.e. there is less than $100 \mathrm{~mW} \mathrm{~m}^{-2}$ of cooling) with or without sub-grid coagulation (see Figs. 8 and S14). 


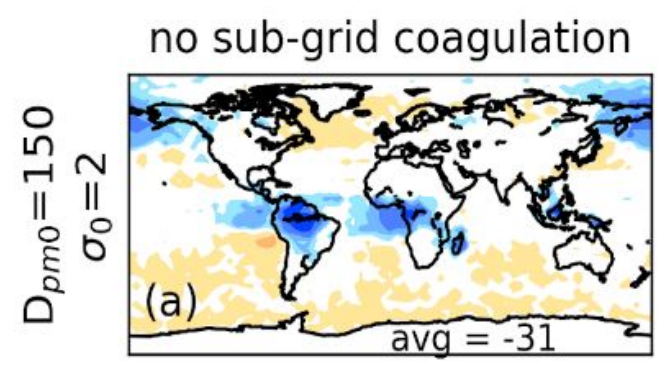

with sub-grid coagulation
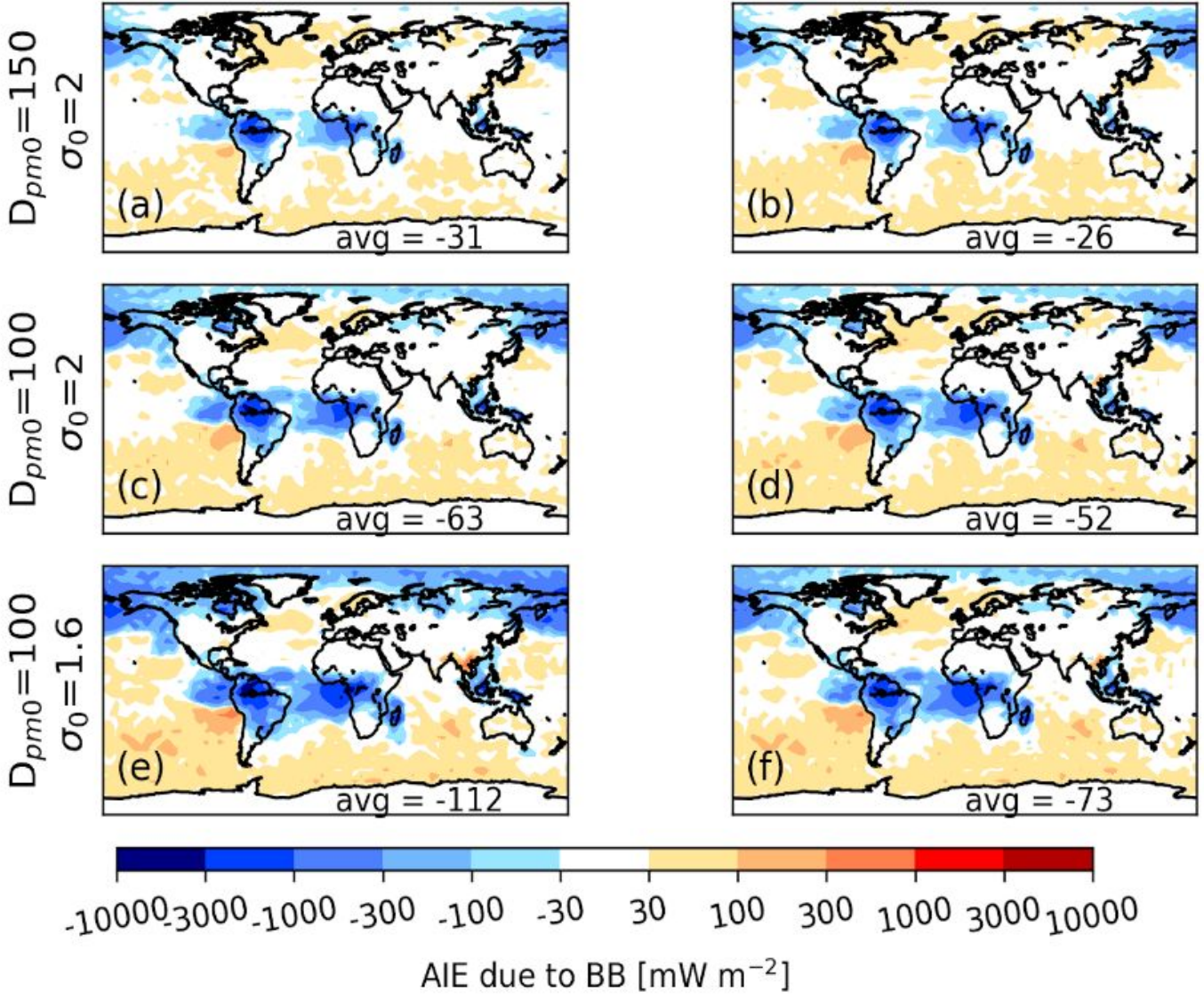

Figure S16: Cloud-albedo aerosol indirect effect (AIE) due to biomass burning aerosols using FINNv1 emissions. Panels (a), (c), and (e) are without sub-grid coagulation (D150_noSubCoag_FINN, noSubCoag_FINN, and s1.6_noSubCoag_FINN, respectively). Panels (b), (d), and (f) are with sub-grid coagulation(D150_SubCoag_FINN, SubCoag_FINN, and s1.6_SubCoag_FINN, respectively). Panels (a) and (b) have an emitted median diameter of $150 \mathrm{~nm}$ and an emitted modal width of 2 . Panels (c) and (d) have an emitted median diameter of $100 \mathrm{~nm}$ and an emitted modal width of 2 . Panels (e) and (f) have an emitted median diameter of $100 \mathrm{~nm}$ and an emitted modal width of 1.6. The number in the bottom right of each panel is the global mean AIE value $\left[\mathrm{mW} \mathrm{m}^{-2}\right]$. 
GFED
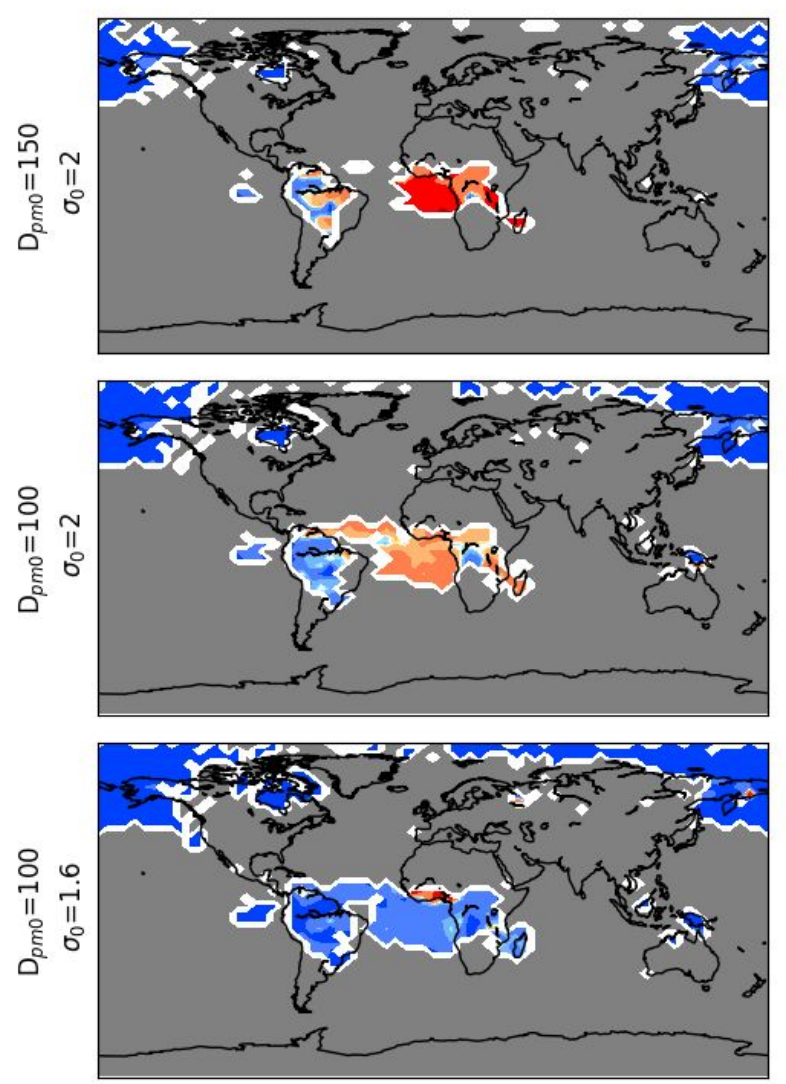

FINN
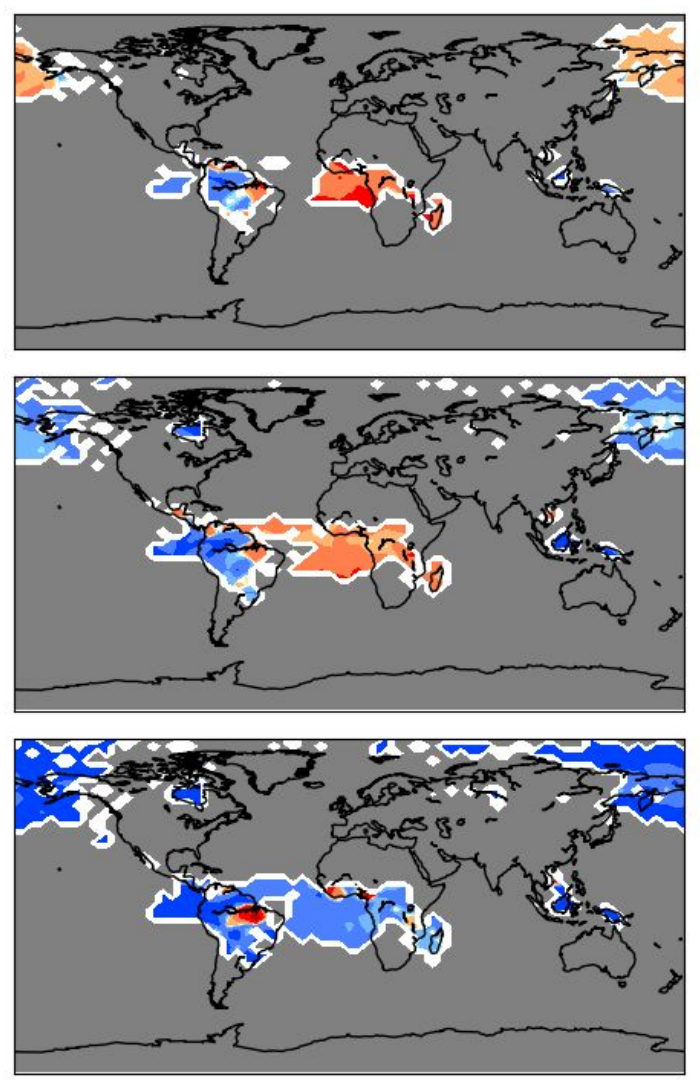

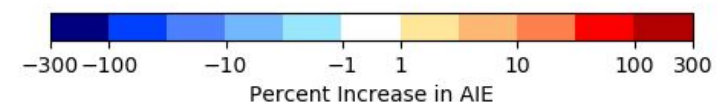

Figure S17: Percent change in cloud albedo aerosol indirect effect (AIE) of biomass burning aerosol due to sub-grid coagulation and GFED emissions (left) or FINNv1 emissions (right) and an assumed in-plume coagulation time of 24 hours. Panels in the top row have an emitted median diameter of $150 \mathrm{~nm}$ and an emitted modal width of 2 . Panels in the middle row have an emitted median diameter of $100 \mathrm{~nm}$ and an emitted modal width of 2 . Panels in the bottom row have an emitted median diameter of $100 \mathbf{n m}$ and an emitted modal width of 1.6. Positive (red) values correspond to an increased cooling tendency of biomass burning AIE due to sub-grid coagulation being added. Regions in grey indicate that the AIE due to biomass burning is a more positive value than $-100 \mathrm{~mW} \mathrm{~m}^{-2}$ (i.e. there is less than $100 \mathrm{~mW} \mathrm{~m}^{-2}$ of cooling) with or without sub-grid coagulation (Figs. 9 and S16). 

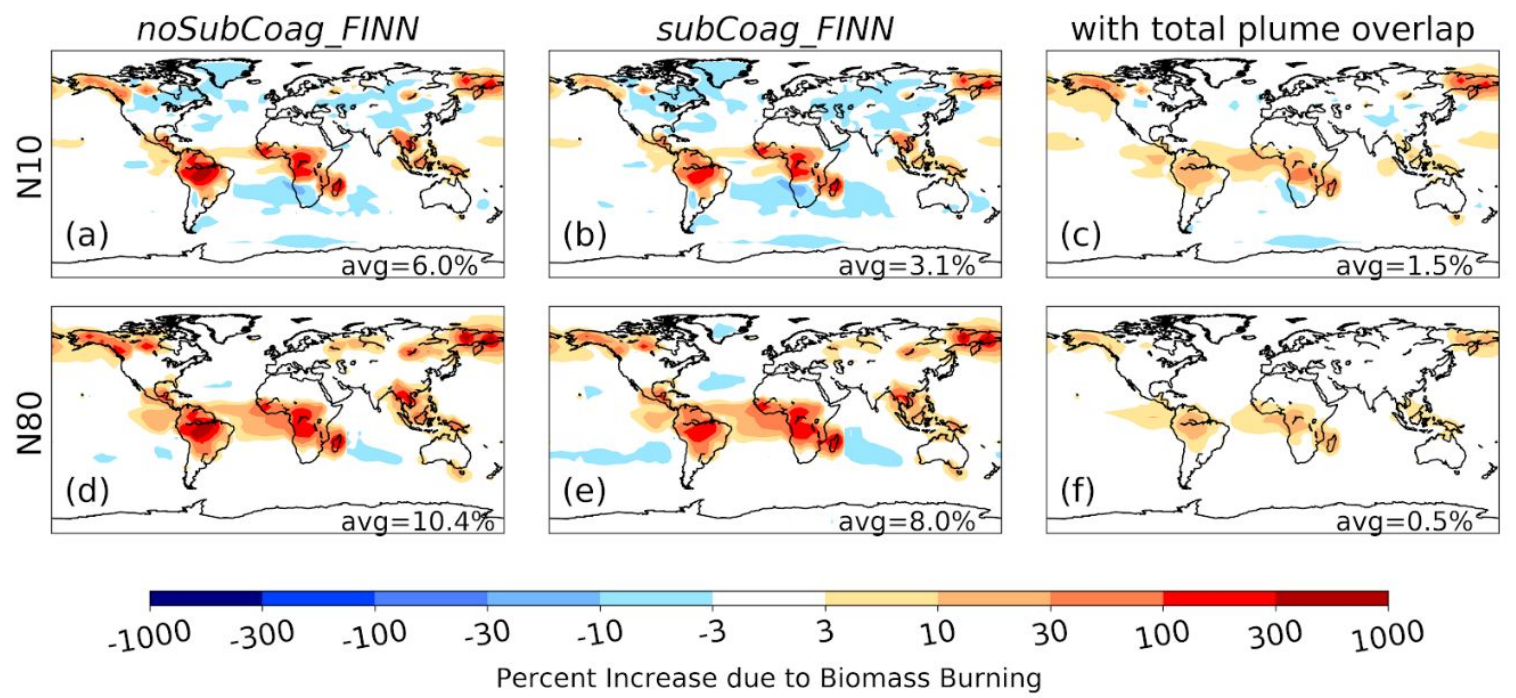

Figure S18: Effect of biomass burning on surface-level N10 (a-c) and N80 (d-f) under three sub-grid coagulation conditions. Panels (a) and (d) show the noSubCoag_FINNcase (no sub-grid coagulation). Panels (b) and (e) show the SubCoag_FINN case (with sub-grid coagulation as in the rest of the paper, where the smoke plumes are treated as without overlap). Panels (c) and (f) show a new case where all smoke plumes in the gridbox completely overlap and form a single "superplume" upon emission into the sub-grid coagulation parameterization. All panels show the percent increase due to FINNv1 biomass burning emissions relative to the $n o B B$ simulation. The number in the bottom right of each panel is the global mean percent increase due to biomass burning. 


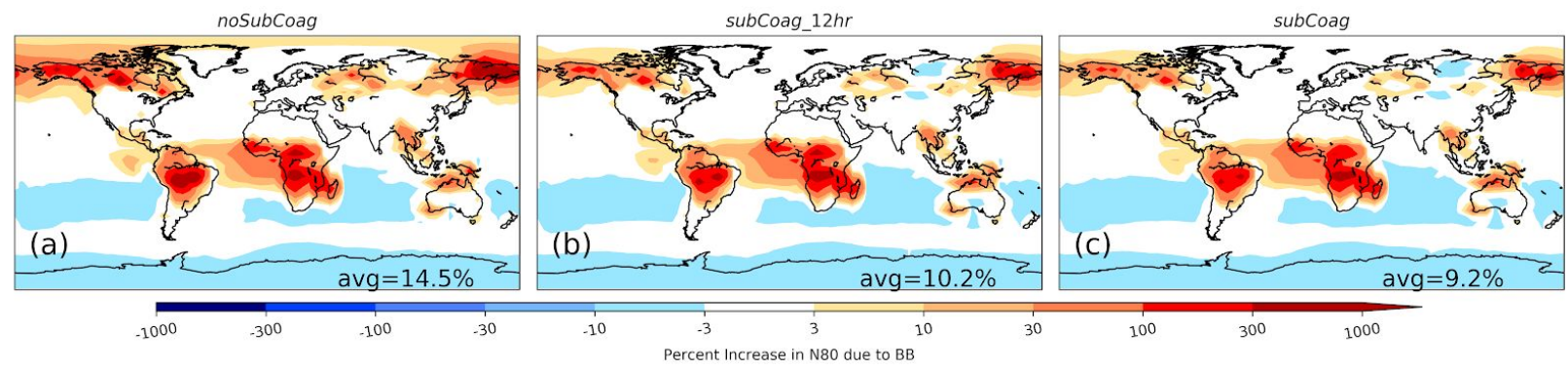

Figure S19: Annual-average percent changes in N80 at the surface level due to the inclusion of GFED biomass burning emissions relative to the simulation without biomass burning (noBB). On the left, there is no sub-grid coagulation (noSubCoag). In the middle, the sub-grid coagulation time is 12 hours (SubCoag_12h). On the right, the sub-grid coagulation time is 24 hours (SubCoag). The number in the bottom right corner of each panel is the global mean percent increase in $\mathrm{N80}$ due to biomass burning. 

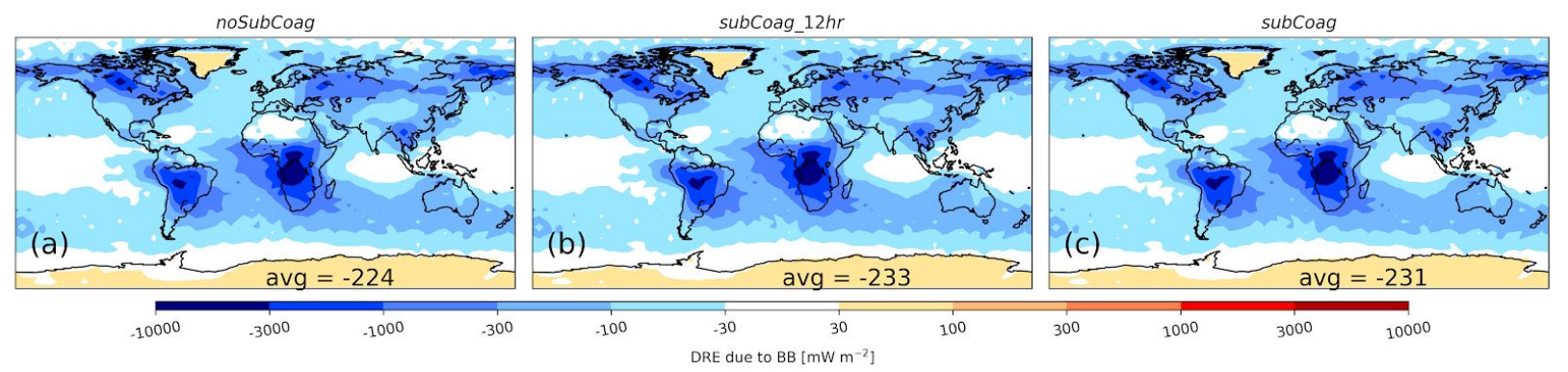

Figure S20: All-sky direct radiative effect due to biomass burning aerosols using GFED emissions and the external-mixing assumption. On the left, there is no sub-grid coagulation (noSubCoag). In the middle, the sub-grid coagulation time is 12 hours (SubCoag_12h). On the right, the sub-grid coagulation time is 24 hours (SubCoag). The number in the bottom right corner of each panel is the global mean value [ $\left.\mathrm{mW} \mathrm{m}^{-2}\right]$. 


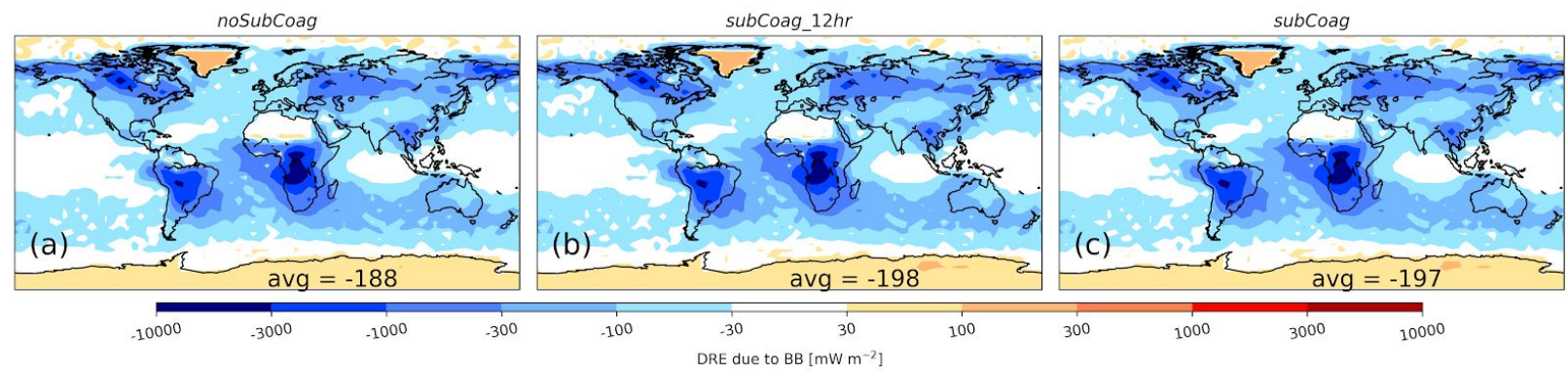

Figure S21: All-sky direct radiative effect due to biomass burning aerosols using GFED emissions and the internal, core-shell mixing assumption. On the left, there is no sub-grid coagulation (noSubCoag). In the middle, the sub-grid coagulation time is 12 hours (SubCoag_12h). On the right, the sub-grid coagulation time is 24 hours (SubCoag). The number in the bottom right corner of each panel is the global mean value [mW $\left.\mathbf{m}^{-2}\right]$. 


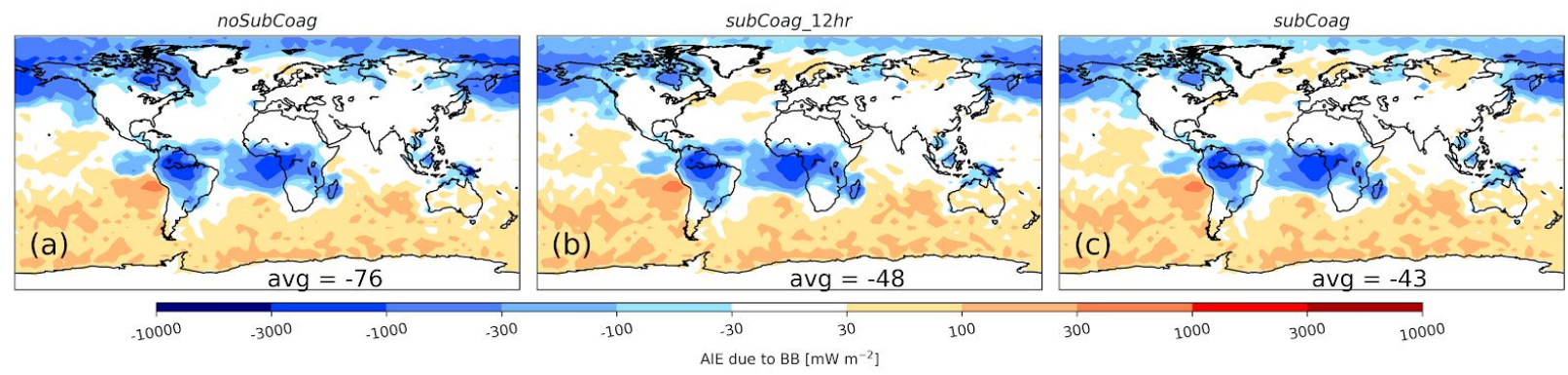

Figure S22: Cloud-albedo aerosol indirect effect due to biomass burning aerosols using GFED emissions. On the left, there is no sub-grid coagulation (noSubCoag). In the middle, the sub-grid coagulation time is 12 hours (SubCoag_12h). On the right, the sub-grid coagulation time is 24 hours (SubCoag). The number in the bottom right corner of each panel is the global mean value $\left[\mathrm{mW} \mathrm{m}^{-2}\right]$. 